EPJ Web of Conferences 61, 02001 (2013)

DOI: 10.1051/epjconf/20136102001

(C) Owned by the authors, published by EDP Sciences, 2013

\title{
The Role of Macroscopic and Microscopic Jet Instabilities
}

\author{
Philip E. Hardee ${ }^{1, a}$ \\ ${ }^{1}$ Department of Physics \& Astronomy, The University of Alabama, Tuscaloosa, AL 35487
}

\begin{abstract}
Relativistic jets, be they Poynting flux or kinetic flux dominated, are current driven (CD) and/or Kelvin-Helmholtz (KH) velocity shear driven unstable. These macroscopic MHD instabilities may be responsible for some of the observed larger scale twisted jet structures and typically do not disrupt jets on less than kiloparsec scales. Here I review our understanding of the jet properties that will lead to the observed relative stability of astrophysical jets. In addition, I review the progress made on the microscopic scale plasma instabilities in shocks and velocity shears that may lead to magnetic field generation and that does lead to the particle acceleration required to produce the observed emission from radio wavelengths to TeV energies. Finally, I discuss these instabilities in the context of the jet in M 87 .
\end{abstract}

\section{Introduction}

Jets are subject to current driven instability (CDI) where toroidal magnetic fields are dominant, and when superAlfvénic are subject to the Kelvin-Helmholtz instability (KHI) driven by velocity shear between the jet and the surrounding medium. Instability is predicted theoretically from the linearized magnetofluid equations, and one might expect stability conditions to provide a tool for selection between different launching and propagation configurations satisfying the magnetofluid equations.

Twisted structures are observed in many AGN jets on sub-parsec, parsec and kiloparsec scales, e.g., 3C 120 [1], $3 \mathrm{C} 273$ [2] and M 87 [3]. The propagation, radial structure and growth or damping of twisted jet structures depends on whether the structure is magnetically (CDI) or kinetically (KHI) induced. Non-relativistic and relativistic jet simulations have shown helical structures attributed to CDI [5-11, 76, 80], or attributed to KHI [13, 111, 113]. Twisted structures and the sense of twist can be induced by precession of the jet base [16], by rotation of the jet fluid, or by the helicity of the magnetic field. It is still not clear whether CDI, KHI or precession is responsible for the observed structures, or whether these different processes operate on different spatial scales.

VLBI techniques and multi-wavelength campaigns probe structures in the deepest parts of AGN jets [17, 18]. VLBI proper motions supply an estimate of the viewing angle, $\theta$, and Lorentz factor, $\gamma$, that link observed timescales and distances to intrinsic timescales and distances. Rotation of the optical polarization vector in some sources suggests helical twisting of the flow and magnetic field between the central engine and the $\mathrm{mm}$ radio core. The observation of moving emission knots indicates sig-

\footnotetext{
ae-mail: phardee@bama.ua.edu
}

nificant macroscopic flow variation ejection events resulting in internal jet shocks.

Blazar emission spectra probe microscopic particle acceleration processes within the jets. In general, the spectrum from radio wavelengths to the $\mathrm{TeV}$ gamma-rays is characterized by two peaks which arise from internal synchrotron and synchrotron self-Compton (SSC) or inverse Compton (IC) processes involving photons from outside the moving zone of emission [19-23]. Fitting spectral energy distributions (SEDs) with one-zone homogeneous electron SSC models to $\mathrm{TeV}$ sources typically requires Doppler factors, $\delta \equiv[\gamma(1-v / c \cos \theta)]^{-1}$, from $10-20$ [24] up to 50 [25] depending on $\mathrm{TeV}$ photon absorption by the IR background. The large Doppler and associated Lorentz factors are problematic [26] because VLBI studies indicate slow moving pc-scale emission features in strong TeV BL Lacs [27, 28]. The slow pc-scale motions led Georganopoulos \& Kazanas (2003) [29] to propose that faster moving material at the jet base relativistically boosts radiation produced by slower moving observed material at the end of an unobserved deceleration zone. Alternatively Ghisellini et al. (2005) [30] proposed a fast jet spine slow jet sheath configuration. These scenarios allow the reduced Doppler factor values indicated by VLBI studies and suggest significant internal longitudinal and/or transverse velocity structure, e.g., shocks and velocity shears, that provide sites for particle acceleration.

In general, rapid non-thermal particle acceleration is required to generate high energy electron synchrotron emitting particles. Fermi acceleration processes [31, 32] require large velocity differences combined with turbulent scattering, e.g., shocks or velocity shear [33]. Fermi or other shock acceleration processes are not likely in the sub-Alfvénic magnetically dominated jet production region from which $\mathrm{TeV}$ flares might originate. In the absence of shock acceleration, acceleration may be provided 
by vacuum gap electric fields in the black hole magnetosphere $[34,35]$, by CDI and magnetic reconnection very close to the central engine [36], or by accretion disk shear and turbulence [37]. High energy synchrotron emission from proton and associated secondary cascades could be important if protons are accelerated to pion production energies in high magnetic fields [35] and for hadronic models (Böttcher, this proceedings).

Rapid observed variability timescales, $\Delta t$, pose severe constraints on the particle acceleration timescale, $\sim \delta \Delta t$ and the emission region size, $\sim \delta c \Delta t$. Correlated $\mathrm{X}$-ray/TeV gamma-ray flares with timescales from 15 minutes (Mrk 421) to a few hours (Mrk $501 \& 1 \mathrm{ES}$ 1959+650) have been observed. Thus, X-ray/TeV flares imply small fast moving "needles-in-a-jet" or "jet-in-a-jet" [39-41, 168] scenarios which provide CDI/reconnection, shock, and velocity shear rapid particle acceleration sites.

\section{Macroscopic Processes}

MHD processes need to be studied from scales on the order of the black hole radius, where magnetic and electric fields likely dominate jet dynamics, out to distances where kinetic effects likely dominate jet dynamics. GRMHD codes are used to study the extraction of rotational energy from a spinning black hole, i.e., Blandford-Znajek mechanism [42, 43], and from the accretion disk [44], i.e., Blandford-Payne mechanism [45]. Simulations [46, 47] show angular momentum transfer in the accretion disk, diffusion of matter and magnetic field inwards, and unsteady outflows near a centrifugally supported "funnel" wall [46]. In general, rotating black hole simulations indicate a Poynting-flux high Lorentz factor spine with $v \sim c$ and $\gamma \sim 10$, and a matter dominated sheath with $v<c$ possibly embedded in a lower speed, $v<<c$, disk/coronal wind. This basic result suggests the fast jet spine and slower jet sheath type structure advocated to reduce the need for very high Lorentz and Doppler boost factors. Note that the Lorentz factor can achieve a maximum in a tenuous boundary layer between an overpressured Poynting-flux spine and a dense low speed kinetically dominated sheath $[48,49]$.

Poynting flux dominated outflows naturally arise on the magnetic field lines threading the horizon of a rotating black hole [34, 45], with acceleration and collimation attributed to the toroidal magnetic field acting in concert with an external confining medium. The strong toroidal magnetic field is CD kink mode unstable [50]. This instability can strongly distort or even disrupt the system. Even so, the outflow may be well collimated by the largescale poloidal magnetic field of the accretion disk [51]. In this case the instability develops in an already collimated flow but the regular structure of the magnetic field can be disrupted via reconnection [50-54]. While gradual acceleration by magnetic forces [55-59] and reconnection of alternating magnetic fields [60-62] have also been proposed as a way to convert Poynting flux to kinetic flux, magnetic energy release via the $\mathrm{CD}$ kink instability is inherent in narrow Poynting dominated jets.

For relativistic jet flow $\gamma_{j}>>1$ implies $E_{\text {kinetic }}>>$ $W_{j} c^{2} \equiv\left\{\rho_{j}+[\Gamma /(\Gamma-1)] P_{j} / c^{2}\right\} c^{2}$. Here $\gamma_{j}$ is the jet Lorentz factor, $\Gamma$ is the adiabatic index, and $W$ is the enthalpy. For a magnetically produced jet we expect the jet speed $v_{j} \sim$ $v_{A l f v e ́ n}$ and $\gamma_{j} \sim \gamma_{A} \sim\left[V_{A} / c\right] \equiv\left[B^{2} /\left(4 \pi W_{j} c^{2}\right)\right]^{1 / 2}>>1$ so there will be relatively low mass loading of the magnetic field lines. Here $B$ is the magnetic field measured in the jet rest frame and the jet is Poynting-flux dominated. In this regime the sound speed is less than the Alfvén wave speed, i.e., $a_{s}^{2} \equiv \Gamma P_{j} / W_{j}<v_{A}^{2} \equiv V_{A}^{2} /\left(1+V_{A}^{2} / c^{2}\right)$. A magnetically dominated jet with a uniform poloidal field component is KH stable when $\gamma_{j}<\gamma_{A}\left[1+\left(W_{j} / W_{e}\right)\left(V_{A, p}^{2} / c^{2}\right)\right]^{1 / 2}$ where $V_{A, p} \equiv\left[B_{p}^{2} /\left(4 \pi W_{j}\right)\right]^{1 / 2}$ and $W_{e}$ is the enthalpy of an unmagnetized external medium. Additional stabilization is provided by the presence of a significantly magnetized sheath or wind even if the jet core is super-Alfvénic [103, 109]. The addition of a toroidal field component is not likely to alter this stability condition and we expect the Poynting-flux jet spine to be KH stable.

\subsection{Current Driven Instability}

A 3D GRMHD simulation performed by [11] indicates non-disruptive kink development and along with VLBI observations suggests that jets are not subject to global disruptive CD kink development. Thus, we are interested in conditions that prevent global disruptive kink development but not necessarily on smaller scales within a jet.

Cylindrical force-free jets are kink stable if the poloidal field is independent of the radius $[65,66]$, but are kink unstable if the poloidal field decreases with the radius $[50,53]$. In a static reference frame or jet confined by rigid walls [67], the Kruskal-Shafranov criterion for instability, $\left|B_{\phi} / B_{p}\right|>2 \pi R / z$, indicates that the instability develops if the length of a static plasma column is long enough for the field lines to go around the column at least once [68]. In the case of non-relativistic rotation, force-free jets are kink unstable if $\left|B_{\phi} / B_{p}\right|>|\Omega| R / c$ [69]. Very recently more general stability analyses including sharp velocity shears have shown that results depend significantly on the magnetic pitch profile and magnetization [70] (Vlahakis, this proceedings). In general, the maximum growth rate increases as the magnetic field strength and helicity increase. In the case of broad velocity shear and toroidal magnetic field short-wavelength CDI velocity shear modes can dominate for sufficiently high shear values [71]. This may provide a means for converting Poynting-flux-dominated jets into matter-dominated jets without global disruptive kink development.

The effects of magnetic helicity, jet density and jet flow profiles can be explored in the non-linear regime via numerical simulations [72-74]. For rigidly moving relativistic configurations with magnetic energy density comparable to or greater than the plasma energy density, [75] found that the kink develops as predicted by linear theory but does not disrupt the force-free helical magnetic field. In the non-linear regime, growth is reduced by reduction in the Alfvén speed and by magnetic helicity decreasing with radius. The characteristic time for the instability to affect strongly the initial structure is $\tau^{s t} \sim 100\left(a / v_{A}\right)$, where $a / v_{A}$ is the Alfvén crossing time across the characteristic magnetic radius, $a$ (approximately the radius at which the 
toroidal field component is a maximum). In the SMBH jet collimation and acceleration environment an Alfvén crossing time is comparable to a light crossing time of the Poynting-flux spine. Jet expansion significantly slows the spatial growth of a kink if it is advected with the jet flow along the expanding jet as the Alfvén crossing time increases with increasing $a$. Jjet rotation also provides a stabilizing influence [76].

Simulations by Mizuno et al. (2011) [77] and this proceedings find that a sub-Alfvénic weakly relativistic velocity shear has profound consequences for kink growth, propagation and the associated flow field. Figure 1 shows how kink growth and the flow field is modified by the location of the velocity shear surface. In these simulations the flow was initialized across the computational grid and perturbed at the inlet. For a velocity shear surface, $R_{j}$, well inside the characteristic magnetic radius, $a$, transverse growth is similar to the static case and the plasma flows through a growing non-moving kink. Ultimately, the flow is disrupted close to the inlet. For a velocity shear surface well outside the characteristic radius, initial growth is similar to that of a static case, the kink is advected with the flow and the flow remains more collimated. For veloc-
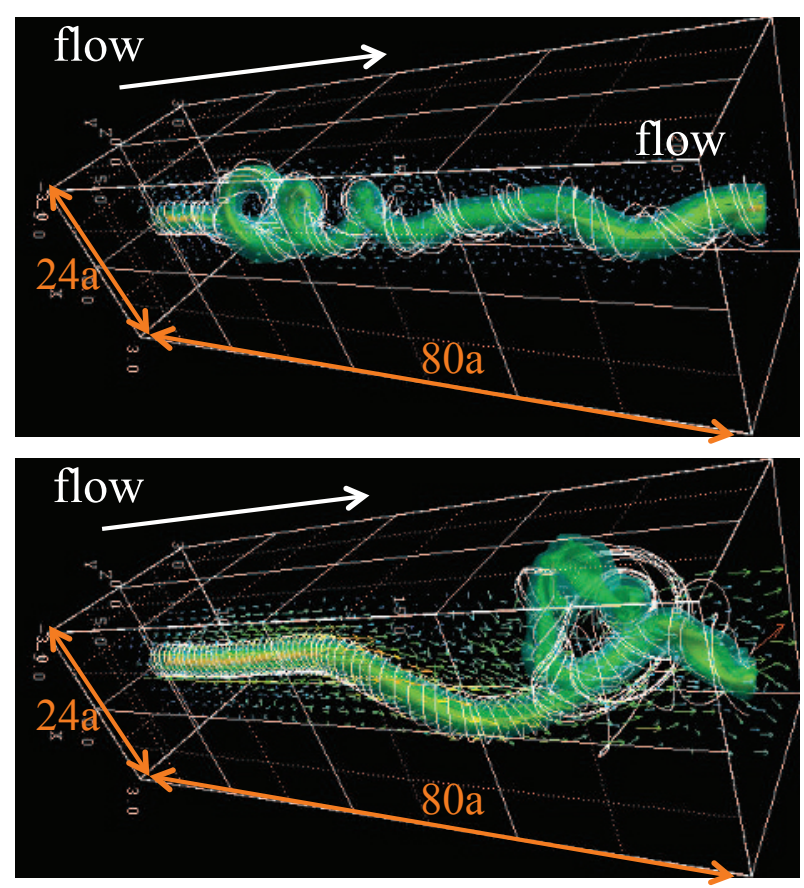

Figure 1. The upper panel shows CDI kink development for a jet with the velocity shear surface, $\mathbf{R}_{j}$, well inside the characteristic radius, $a$, and flow is through a stationary growing kink. The lower panel shows CDI kink development for a jet with the velocity shear surface, $\mathbf{R}_{j}$, well outside the characteristic radius, $a$, and the kink is advected with the flow. White lines show the magnetic field and the flow field is indicated by the arrows.

ity shear radii on the order of the characteristic radius the growth rate is slower than for the static case, the kink propagates more slowly than the flow, slows further as transverse growth approaches the velocity shear surface, and there is some flow through the moving kink.
For a CDI kink advected with the jet flow, the "temporal" growth rate $\omega_{i} \propto\left(\gamma_{j} a\right)^{-1}$ [53], we expect growth lengths $\ell_{e} \propto \gamma_{j} a$, and the length for the instability to strongly effect the initial structure becomes $z \gtrsim \gamma_{j} \tau^{s t} v_{j} \sim$ $100 \gamma_{j} a$. The spatial growth of the kink mode will be relatively slow for a typical jet with opening angle $\theta \sim \mathrm{few} / \gamma_{j}$ ([67]). However, if the kink moves more slowly, present results suggest a temporal development scaling like $\tau \sim$ $\gamma_{k}^{\alpha} \tau^{s t}$ with $3 \geq \alpha \geq 1$ for a moving kink with $R_{j} \gtrsim a$. In this case the condition for the instability to affect the jet structure might be written as $z>100 \gamma_{k}^{\alpha}\left(v_{k} / c\right) a$, where $\tau^{s t} \equiv 100(a / c)$ and $0<v_{k} \leq v_{j}$ is a function of $R_{j} / a$ that is sensitively dependent on the location of the velocity shear surface provided $R_{j} / a<<10$. Spatial growth simulations, e.g., Figure 1, show significant stabilization of the moving kink relative to the stationary kink.

A high speed jet spine containing a uniform poloidal and decreasing toroidal magnetic field confined by a denser slower moving more weakly magnetized sheath will be relatively stable. Thus, disruptive CDI and conversion of Poynting to kinetic flux would occur only on small scales within the broader jet flow.

\subsection{Kelvin-Helmholtz Instabiity}

Non-relativistic analytical work [78, 79] and numerical simulations [80, 81] show that CDI growth rates exceed KHI growth rates for sufficient magnetic field strength and helicity. This work appears confirmed for relativistic flows [70] and Vlahakis (this proceedings). However, conversion of magnetic to kinetic energy seems likely within no more than a few 10s of parsecs from the central engine, and jets now are unstable to the KHI. KHI induced vorticies lead to entrainment, and ultimately can lead to disruption of the collimated flow. In the case of spine-sheath flows, entrainment into the high speed spine can lead to mixing of spine and sheath in only 200 jet radii [82]. Jet generation simulations along with observations and modeling, e.g., Blazar SEDs and the transverse structure of optical and radio polarization along with the proper-motion structure of the M 87 jet, suggest the existence of a high speed spine out to distances of at least hundreds of parsecs, and, of course, jet collimation in some sources is observed out to hundreds of kpc. Thus, relativistic jets must have configurations in which the KHI and associated mixing are partially suppressed.

The KHI properties of jets have been reviewed in various astrophysical contexts [83-89]. Theoretical analyses have been performed for fluid jets with sharp boundaries [90-94], with a velocity shear layer [95], or with rotation [96]. Additional investigations considered magnetic fields parallel to the flow, e.g., [97-103], and considered toroidal magnetic fields [104-106]. Relativistic jet simulations have found growth rates [107-109], and jet structure [110-113], predicted theoretically.

KHI induced jet structures consist of pinch, helical, elliptical, etc. "normal mode" jet distortions [91]. Each normal mode formally consists of a fundamental (pinch) or surface (helical, elliptical, etc.) and accompanying body solutions with different radial structure (see Fig. 5 in [86]; Fig. 13 in [13]; Fig. 7 in [111]). Helical, elliptical, etc. 
surface modes produce high pressure helically twisted filaments and corrugations to the jet's surface, while the accompanying body modes produce helically twisted high pressure filaments in the jet's interior. Spatial growth lengths scale with the Lorentz factor, magnetosonic Mach number and jet radius. In the subsonic but super-Alfvénic limit the body modes are stable. Pinch structures can be triggered by flow variation or jet overpressure [112], and twisted structures can be induced by precession, rotation, or magnetic helicity.

On astrophysical jets we expect velocity shears to develop between the jet spine and sheath and the external medium. This shear layer significantly modifies the growth of wavelengths comparable to the shear layer thickness [95, 114-116]. There can be enhanced instability at short shear layer resonant wavelengths but, in general, higher order surface and body modes are stabilized or growth rates reduced by the presence of velocity shear $[117,118]$. Shear layer resonances result in heating of the shear layer in numerical simulations and serve to significantly reduce mixing.

Large scale helical and elliptical distortion are not suppressed by the development of a velocity shear layer [95] and ultimately large scale helical and to a lesser extent elliptical distortion will lead to catastrophic mixing and disruption of collimated jet flow [119]. However, the reduction of spatial growth of large scale distortion by larger Lorentz factor, magnetosonic Mach number will allow jets to remain collimated to larger distances. Further stabilization is provided by jet expansion. Along expanding jets a growing long wavelength distortion can be advected down the expanding jet into a shorter wavelength regime. Figure 2 shows the difference between a perturbation that generates a long helical wavelength and one that generates a short helical wavelength. In both simulations the jet was

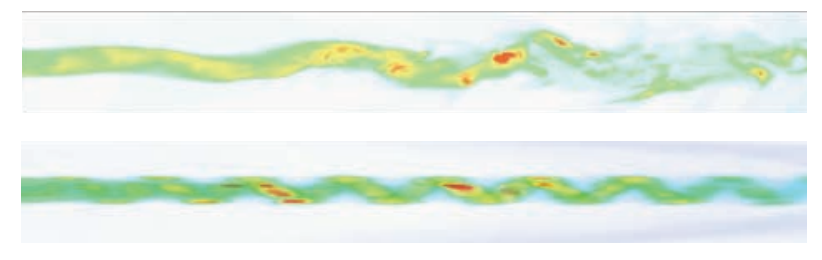

Figure 2. The upper panel shows a 2D image of KHI long helical wavelength development and the lower panel shows a 2D image of KHI short helical wavelength development.

established across the computational grid and precessionally perturbed at the origin. The shorter wavelength saturates at an amplitude that does not lead to significant surface distortion and mixing [120].

Additionally, equipartition or weaker magnetic fields are capable of stabilizing a shear profile through non-linear saturation [121-124]. Figure 3 shows the effect of poloidal and helical equipartition magnetic fields on KHI development and mixing [119]. In both simulations the jet was established across the computational grid and precessionally perturbed at the origin. A similar large scale helical twist develops in both simulations as a result of the precession. The simulation containing a poloidal magnetic field de- velops elliptical and other surface mode distortions nearly identical to a purely fluid simulation and shows evidence for considerable mixing as a result of these distortions. In the simulation containing a helical magnetic field surface distortions and mixing are suppressed because the toroidal magnetic field component inhibits development of surface corrugations. In stronger magnetic fields mode-mode in-
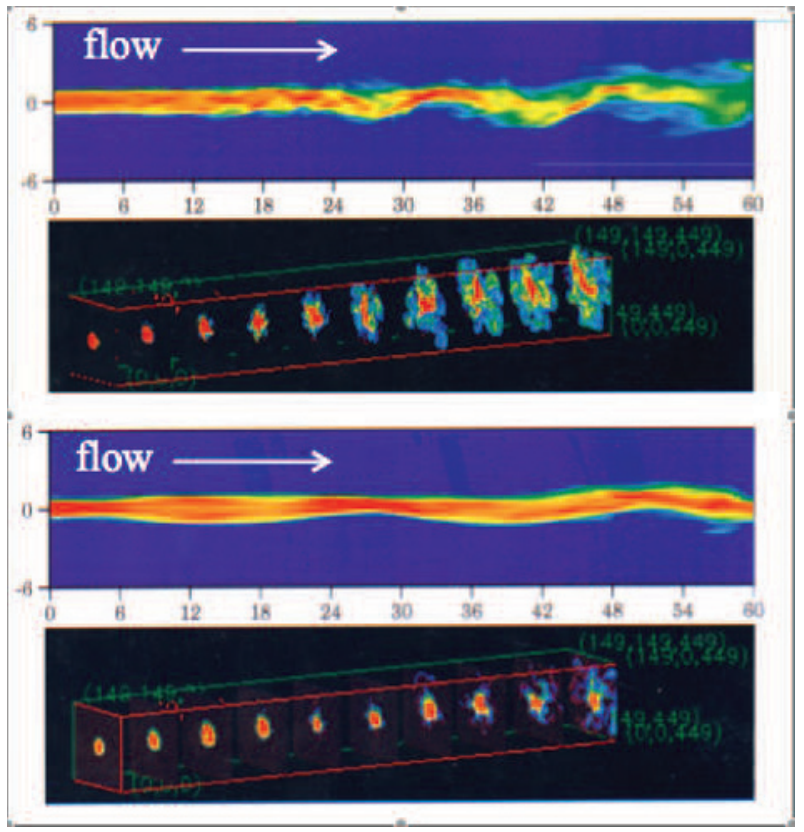

Figure 3. The upper two panels show a 2D image and cross sections of KHI development for a jet with an equipartition poloidal magnetic field. The lower two panels show a 2D image and cross sections of KHI development for a jet with an equipartition helical magnetic field.

teraction between KHI and CDI inhibits development of $\mathrm{KH}$ vorticies and mixing [81]. While mixing can never be completely suppressed, the effects of mixing will be minimized if the jet's spine-sheath core entrains only lighter material, e.g., from a hot tenuous shear layer or the cocooning hot tenuous shocked jet material associated with FR II type radio sources.

In general, when a jet initially becomes super-Alfvénic and $\mathrm{KH}$ unstable, interaction between the CDI and the KHI can be stabilizing. As magnetic fields decline further and KHI dominates CDI, theory and simulations [103, 109] indicate that relatively strong magnetic fields combined with a spine-sheath structure can slow the spatial growth of the KHI modes. Magnetic helicity along with advection down an expanding jet lead to additional stabilization and reduction in mixing. Thus, it should be possible for a jet to maintain a spine sheath structure and ordered helical magnetic field considerably beyond the sub- to superAlfvénic transition. At large distances where the flow is super-magnetosonic, KHI growth and mixing are still reduced by weaker equipartition magnetic fields, wave advection down an expanding jet and the presence of a hot tenuous shear layer or cocoon. All of these different effects in concert allow relativistic jets to maintain an organized spine-sheath structure to distances of at least 100s of par- 
secs and also remain collimated to the observed hundreds of kpc distances.

\section{Microscopic Processes}

Inside the black hole magnetosphere and in the near jet generation zone where magnetic fields are large and flows are sub- to trans-Alfvénic, the dominant particle acceleration processes should be associated with vacuum gap electric fields [125-127] and also with CDI driven magnetic reconnection.

When the flow is super-Alfvénic or supermagnetosonic (location depending on magnetic reconnection at various scales, associated particle heating/acceleration and mass loading of the Poynting-flux spine) particles can be accelerated via CDI driven reconnection, and in shocks via the filamentation instability, Fermi processes and reconnection, and in velocity shears via the kinetic Kelvin-Helmholtz instability (KKHI).

Microphysical investigation of these particle acceleration processes can provide the electron energy spectrum, the fraction, $\epsilon_{B}$, of the energy density in the plasma contained in the magnetic field, and the fraction, $\epsilon_{e}$, of the plasma kinetic energy transferred into relativistic electrons.

\subsection{Relativistic Shocks: Filamentation Instability \& Reconnection}

The microphysics of relativistic shocks is being investigated using relativistic particle-in-cell (RPIC) codes [128, 129] and theoretically [130, 131]. In relativistic shocks the most rapid particle acceleration is provided in situ by the filamentation instability, so named as the interpenetrating plasmas form the current filaments and associated magnetic fields shown in Figure 4.

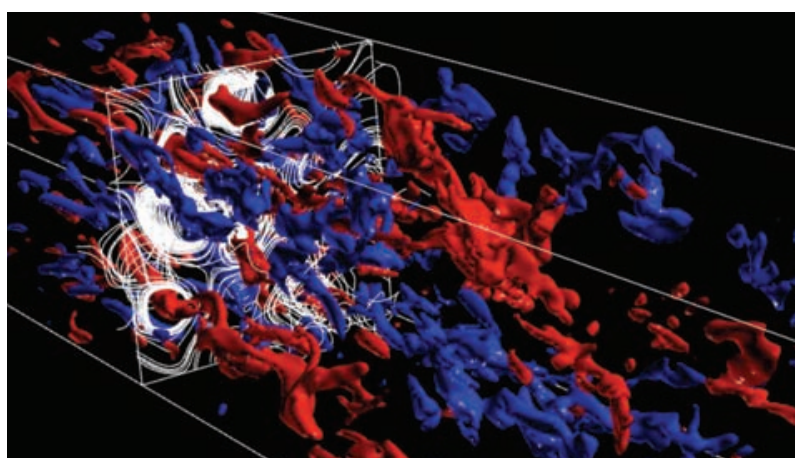

Figure 4. Positive (blue) and negative (red) current filaments associated with a Lorentz factor 15 relativistic pair shock. The current generated (white) magnetic field lines are shown in a 2D slice parallel to the shock plane.

Un-magnetized three-dimensional (3D) particle-incell (PIC) shock simulations of relativistic pair jets penetrating an ambient pair plasma [129] shown in Figure 5 show the development of a propagating hydrodynamiclike trailing shock, contact discontinuity, and leading shock system associated with a Lorentz factor 15 jet. The lack of initial magnetic field in the jet and ambient medium in this hydrodynamic shock system allows jet and ambient particles to interpenetrate. Jet particles begin to thermalize in interaction with those thermalized ambient particles not swept up at the contact discontinuity. On average jet particles are slowed and thermalized further at the trailing shock but some jet particles continue downstream at nearly lightspeed to form a leading edge where interaction with the ambient particles begins. Ambient particles are accelerated and thermalized at the leading shock. In the contact discontinuity region between leading and trailing shocks, jet and ambient particles move on average with the contact discontinuity.

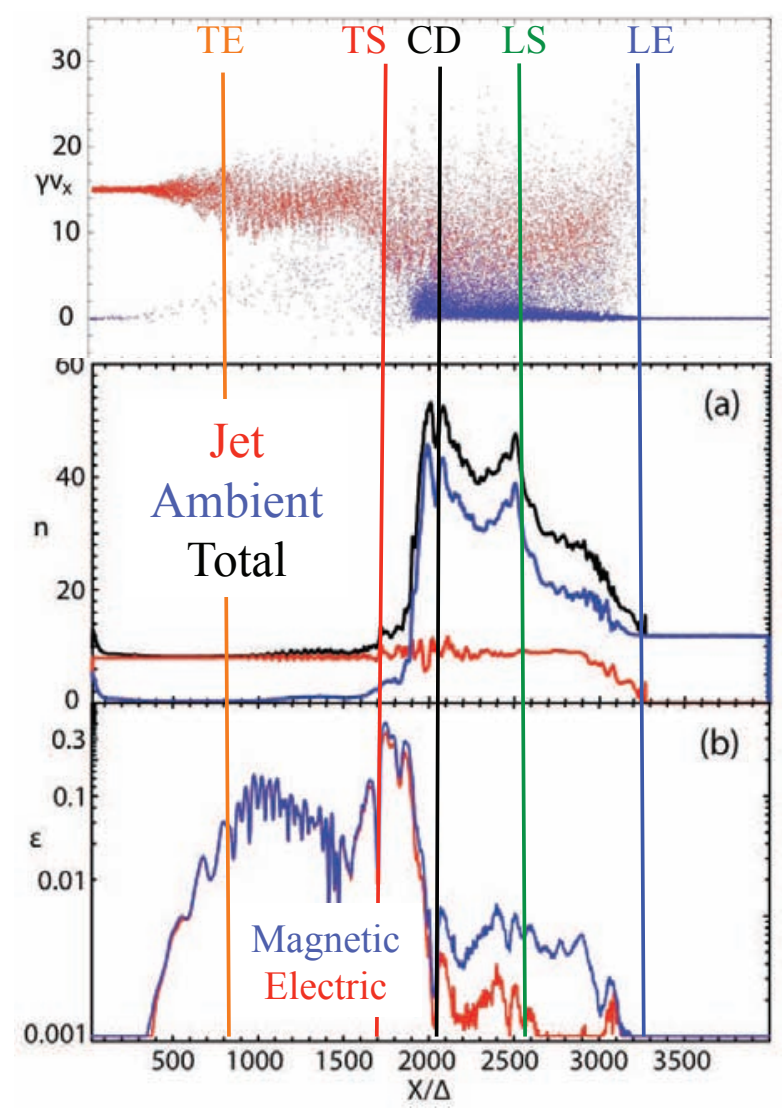

Figure 5. The upper panel is a velocity, $\gamma v_{x}$, space, $x$, plot of individual jet (red) and ambient (blue) electrons in the pair plasma shock system. The middle panel shows jet (red), ambient (blue) and total (black) particle number densities in the pair plasma shock system. The lower panel shows the magnetic (blue) and electric (red) field energy densities. The magnetic and electric field energy densities reach $30 \%(\epsilon=0.3)$ of the total (thermal plus kinectic) plasma energy density in the trailing shock. The vertical lines indicate the locations of the trailing edge (TE) at which interaction begins, the trailing shock (TS), the contact discontinuity (CD), the leading shock (LS) and the leading edge (LE) associated with the fastest moving jet particles.

Pair plasma shocks have been investigated in the contact discontinuity frame over a broad range of magnetic obliquities [132]. When magnetic fields are introduced into the jet and ambient media, the interpenetration of jet and ambient particles seen in Figure 5 is greatly reduced. Both filamentation and Fermi type particle acceleration is observed with particle acceleration and non-thermal spectral index sensitive to the magnetic obliquity. Other devel- 
opments include the self-consistent computation of emission from pair plasma shocks [133]. A 2D based selfconsistent pair plasma simulation result carried out in the reference frame of the shock contact discontinuity reveals synchrotron like self-absorbed spectra at lower frequencies, a thermal component, and higher frequency suprathermal emission over a six order of magnitude frequency range. A 3D self-consistent computation of pair plasma shock emission from a propagating shock system shows that the spectral peak emissivity and frequency rise as the jet Lorentz factor and temperature increase [134, 135] and (Nishikawa et al., this proceedings).

Recent simulations in the contact discontinuity frame (a single shock is formed by reflecting the inflowing plasma off a rigid wall) have studied the effect of increasing the positive particle mass. There are significant differences in the microphysical shock structure when compared to pair shocks at comparable magnetic field strengths and directions that result from the different positive and negatively charged particle inertia [136]. In these simulations, only weak magnetizations parallel to the shock surface seem to provide efficient acceleration to maximum energies [137]. Choi et al. (in preparation) have performed 3D particle-in-cell (PIC) propagating shock simulations that find sharper features than the previous pair propagating shock simulations including more efficient electron acceleration in addition to ion acceleration in the shock transition regions. Results are different from previous ionelectron contact discontinuity simulations due to the dual shock and contact discontinuity structure. The total magnetic field energy decreases but higher electromagnetic fields extend over a spatially extended dual shock transition region.

Additional particle acceleration associated with shock driven reconnection has been demonstrated by Sironi \& Spitkovsky [138]. In the simulation, alternating fields in a relativistic striped wind are annihilated in a shock by magnetic field reconnection that led to power law particle acceleration in the reconnection electric field. Ultimately the differences between strongly and weakly magnetized pair and electron-ion shocks including reconnection will provide estimates of the shock strength, magnetization and composition that will yield appropriate electron and/or cosmic ray energy spectra.

\subsection{Relativistic Velocity Shear: Kinetic Kelvin-Helmholtz Instability}

Velocity shears can play an important role in particle acceleration scenarios, since these shears trigger the KKHI. Counter-streaming simulations show that the KKHI leads to magnetic field generation and particle acceleration [139, 140]. As KKHI grows the electrons from the counterstreaming flows cross the shear-surface. DC current sheets form around the shear-surface and induce a DC component in the magnetic field as shown in Figure 6. This DC magnetic field is not captured in MHD, but a kinetic treatment fully captures the generated field structure and the particle acceleration.

Recent work by Nishikawa et al. ([141] and this proceedings) uses a setup in which a relativistic jet plasma is
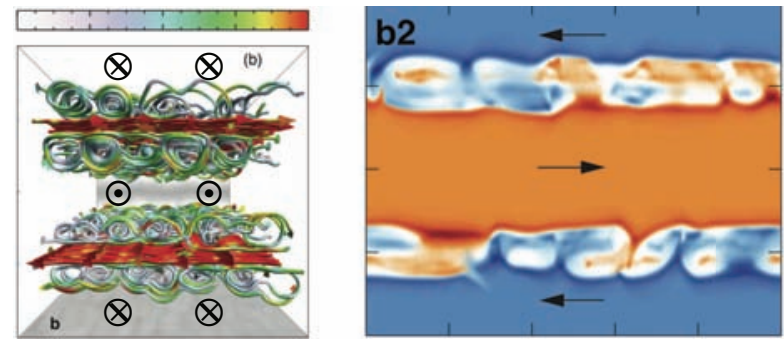

Figure 6. The left panel shows the magnetic field line structure and stength (red to grey) around the velocity shear surfaces with jet flow out of the page bounded by counter-streaming flow into the page. The right panel shows electron density structure in a 2D slice along the flow direction with flow indicated by the arrows. Adapted from Alves et al. [139].

surrounded by a stationary or moving sheath plasma. Initial growth of the KKHI is via an electrostatic 2-stream instability. Figure 7 shows a numerical solution of the electro-static dispersion relation (see Alves dissertation eq. 3.29, and [141]). Analytic solutions to the disper-

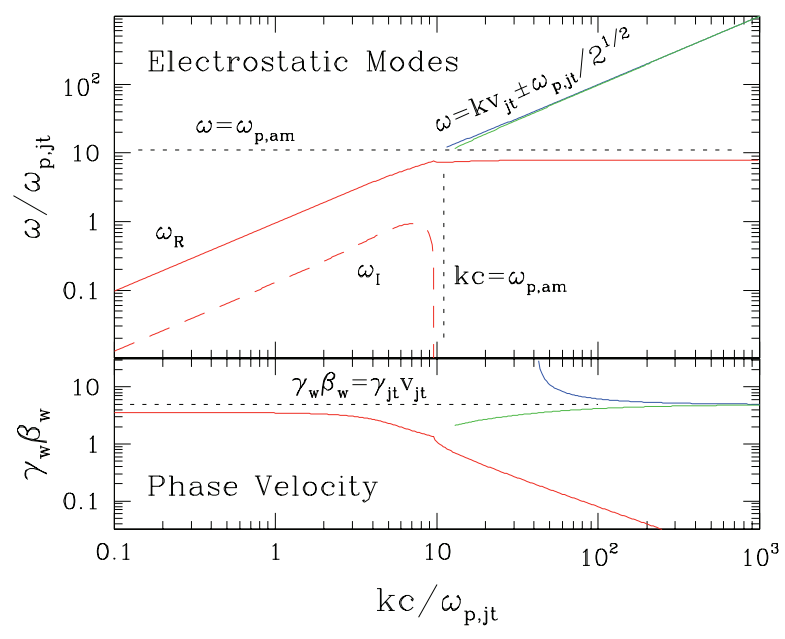

Figure 7. Exact dispersion relation solutions for a test case using the parameters, $n_{\mathrm{jt}}=n_{\mathrm{am}}=0.1 \mathrm{~cm}^{-3}, V_{\mathrm{jt}} / c=0.979796$ with $\gamma_{\mathrm{jt}}=5.0$, and $V_{\mathrm{am}}=0$. The upper panel shows electrostatic solutions as a function of wavenumber, $\mathrm{k}$, parallel to the flow. The real part, $\omega_{\mathrm{R}}$, and imaginary part, $\omega_{\mathrm{I}}$, of the frequency are indicated by the solid and dashed lines, respectively. The lower panel shows the phase velocity, $\gamma_{\mathrm{w}} \beta_{\mathrm{w}}$, where $\beta_{\mathrm{w}} \equiv \omega_{\mathrm{R}} / k c$.

sion relation are available in the low and high wavenumber limits. In the high wavenumber limit with $V_{\mathrm{am}}=0$, $\omega \simeq k V_{\mathrm{jt}} \pm \omega_{\mathrm{p}, \mathrm{jt}} / \sqrt{2}$, and in the low wavenumber limit:

$$
\begin{aligned}
\omega & \simeq \frac{\left(\gamma_{\mathrm{am}} \omega_{\mathrm{p}, \mathrm{jt}} k V_{\mathrm{am}}+\gamma_{\mathrm{jt}} \omega_{\mathrm{p}, \mathrm{am}} k V_{\mathrm{jt}}\right)}{\left(\gamma_{\mathrm{am}} \omega_{\mathrm{p}, \mathrm{jt}}+\gamma_{\mathrm{jt}} \omega_{\mathrm{p}, \mathrm{am}}\right)} \\
& \pm i \frac{\left(\gamma_{\mathrm{am}} \omega_{\mathrm{p}, \mathrm{jt}} \gamma_{\mathrm{jt}} \omega_{\mathrm{p}, \mathrm{am}}\right)^{1 / 2}}{\left(\gamma_{\mathrm{am}} \omega_{\mathrm{p}, \mathrm{jt}}+\gamma_{\mathrm{jt}} \omega_{\mathrm{p}, \mathrm{am}}\right)} k\left(V_{\mathrm{jt}}-V_{\mathrm{am}}\right),
\end{aligned}
$$

where $\omega_{\mathrm{p}, \mathrm{jt}}$ and $\omega_{\mathrm{p}, \mathrm{am}}$ are the plasma frequencies $\left(\omega_{\mathrm{p}}^{2} \equiv\right.$ $\left.4 \pi n e^{2} / \gamma^{3} m_{\mathrm{e}}\right)$ of jet and ambient electrons, respectively, $k$ is the wave number parallel to the jet flow, and $\gamma_{\mathrm{jt}}$ and $\gamma_{\mathrm{am}}$ are Lorentz factors of jet flow electrons, $V_{j t}$, and ambient flow electrons, $V_{a m}$, respectively. Here the real part gives 
the phase velocity and the imaginary part gives the temporal growth rate and directly shows the dependence of the growth rate on the velocity difference across the shear surface.

The structure and global evolution of the strong transverse magnetic field generated by the KKHI in the jetsheath configuration is different from that of the counterstreaming flows. In the jet-sheath configuration the major components of growing modes are $E_{\mathrm{z}}$ perpendicular to the flow boundary, and $B_{\mathrm{y}}$ transverse to the flow direction. After the $B_{\mathrm{y}}$ component is excited an induced electric field $E_{\mathrm{x}}$ parallel to the flow direction becomes larger, while other components remain very small. Surprisingly, the structure and growth rate of KKHI for ion/electron mass ratios $m_{\mathrm{i}} / m_{\mathrm{e}}=1836$ and $m_{\mathrm{i}} / m_{\mathrm{e}}=20$ are similar. This indicates that ion dynamics not captured using the real mass ratio on short simulation time and length scales do not modify the basic result.

The jet sheath configuration is similar in spirit, although not in scale, to that proposed for active galactic nuclei (AGN) relativistic jet spines surrounded by a slower moving sheath, and is particularly relevant to the "jetin-a-jet" or "needles-in-a-jet" scenarios where small scale high speed structures lie within a more slowly moving jet plasma. Work in progress (Nishikawa et al., this proceedings) will address the particle acceleration and radiation that accompany KKHI.

\section{The Case of M 87}

M 87 (Virgo A, NGC 4486, 3C 274), a giant elliptical galaxy near the center of the Virgo Cluster, is a FanaroffRiley Type I source, the spectral energy distribution (SED) is similar to that of an LBL, and this suggests that M 87 is a misaligned BL Lac. The well-known kpc-scale jet in this galaxy is a prominent source of radio, optical, near-ultraviolet (NUV) and X-ray emission. On projected scales of $\sim 0.1-1 \mathrm{kpc}$, the bright knots, the filamentary emission between the knots, and the bends and twists in

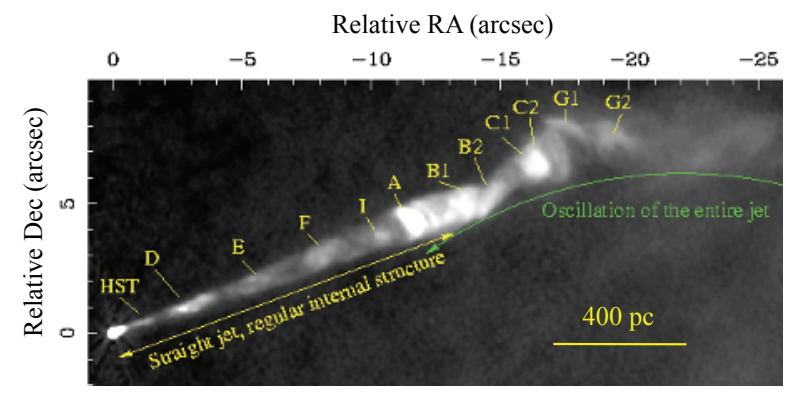

Figure 8. VLA image taken at $15 \mathrm{GHz}$ with resolution 0.15 arcsec and with prominent features labeled. Length scale is in the sky plane.

the jet can easily be identified in the radio image shown in Figure 8 [142] and also in the optical [143] and NUV [144]. While X-ray images from Chandra are not of comparable resolution, the overall jet structure is similar [144].

Relativistic proper motions constrain the viewing angle, $\theta$. The fastest observed optical motion of $\beta^{\mathrm{ob}}=$ $6.1 \pm 0.6[145]$ is at HST-1, and requires that $\theta<19^{\circ}$.
The fastest observed radio motion of $\beta^{\mathrm{ob}}=4.3 \pm 0.7$ [146] is also at HST-1. At a viewing angle of $\theta=15^{\circ}$, the optical and radio motions indicate Lorentz factors of $\sim 7.5$ and $\sim 4.5$, respectively, and suggest a spine-sheath structure at HST-1. In general, the optical/NUV jet is more concentrated in the knots and towards the jet axis than the radio jet, and along with the optical and radio polarizations suggest a spine-sheath structure along the kpc-scale jet [147]. In particular, the more edge brightened radio jet suggests particle acceleration in a velocity shear layer. In general, radio motions are less than optical motions along the kpcscale jet, and both imply jet deceleration beyond HST-1 [148]. Optical, NUV and X-ray emission along the kpcscale jet require in situ particle acceleration within the jet as travel times at lightspeed along the jet are much longer than the synchrotron radiative lifetime [149].

\subsection{VLBA Observations \& Implications}

At a distance of $16.7 \mathrm{Mpc}$ [150], 1 mas $\sim 0.08 \mathrm{pc}$, the M 87 black hole has a mass of $(6.1 \pm 0.4) \times 10^{9} \mathrm{M}_{\odot}$ [151], and 1 mas $\sim 142 \mathrm{R}_{S}$ where $\mathrm{R}_{S} \equiv 2 G M / c^{2} \sim 1.8 \times 10^{15} \mathrm{~cm}$. VLBA observations of the jet with $\sim 0.3$ mas resolution probe projected sizes down to $\approx 50 \mathrm{R}_{S}$. For an assumed viewing angle of $\theta=15^{\circ}$, VLBA resolution along the jet is $\approx 230 R_{S}$ and HST- 1 is $\approx 5.3 \times 10^{5} R_{S}$ along the jet.

The VLBA observed M 87 at $43 \mathrm{GHz}$ roughly every three weeks $[152,153]$ in 2007. An averaged jet image based on 23 images from the VLBA monitoring project is shown in Figure 9. The image shows limb brightening

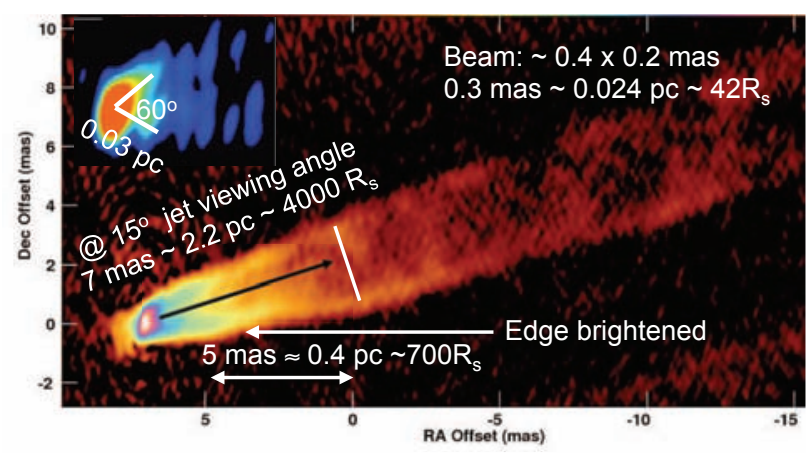

Figure 9. Averaged image of the jet and counter-jet at $43 \mathrm{GHz}$. The faint structure to the left of the radio core is the previously detected counter-jet $[154,155]$. The insert at the upper left is from Junor et al. [156] and indicates an apparent opening angle of $60^{\circ}$ close to the core.

like the kpc jet to within 0.5 mas of the radio core $\left(270 R_{S}\right.$ along the jet) and a faint counter-jet. The inner jet structure beyond about 7 mas $\left(2.2 \mathrm{pc}\right.$ at $\left.\theta=15^{\circ}\right)$ is similar to the radio structure from $10 \mathrm{pc}$ to $\mathrm{kpc}$ scales $[148,157]$.

Transverse slices of the intensity image separated by about half a beamwidth were made along the jet and counter-jet axis. The intensity along the jet edges is typically twice the intensity at jet center. The intensity slices were used to obtain the jet width shown in Figure 10 (Walker et al., in preparation). Not shown is the counterjet side but symmetry in the width on either side of the core along with similar edge-brightening confirm the identification of the faint structure with a counter-jet, and place the 


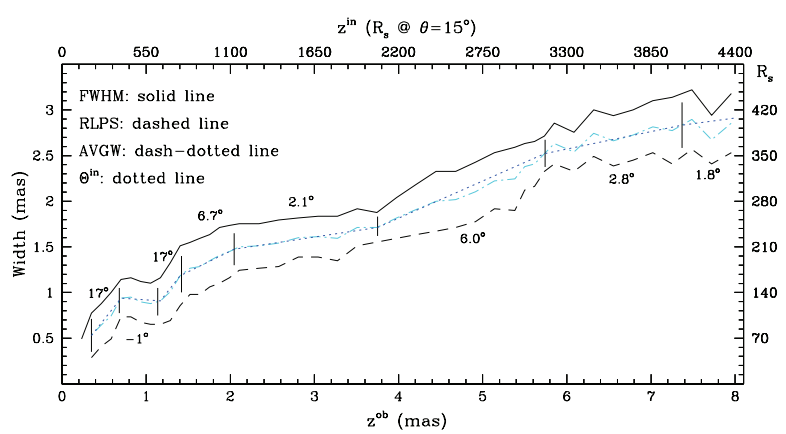

Figure 10. Jet width from full width at half maximum (FWHM) and from the separation of the jet edge intensity peaks, ridgeline peak separation (RLPS). An average (AVGW) of these two widths is shown along with the associated full intrinsic opening angle between the vertical lines assuming a $15^{\circ}$ viewing angle. Width and intrinsic distance down the jet in units of $R_{S}$ are shown on the right and top scales, respectively.

radio core to within about 0.06 mas (half the slice spacing) of the central engine. An initial intrinsic opening angle of $\Theta^{i n}=17^{\circ}$ allows a part of the jet flow to be pointed $\leq 7^{\circ}$ to the line of sight. Collimation features are evident at $\sim 1$ mas and $\sim 4$ mas but there is no evidence in the intensity image for shocks at these locations.

At $\theta=15^{\circ}$, the observed intensity ratio (not shown) between jet and counter-jet implies acceleration from $\beta \sim$ 0.5 to $\sim 0.7$ between $z^{o b} \sim 0.6$ mas and $\sim 1.3$ mas $\left(\sim 385 \mathrm{R}_{S}\right.$ and $\left.\sim 715 \mathrm{R}_{S}\right)$. This result is consistent with motions of $\sim c / 2$ found close to the core with motions up to about $\sim 2 c$ at distances beyond $\sim 1$ mas (Mertens $\&$ Lobanov, this proceedings). These results suggest an increase from subluminal to superluminal motion at the $\sim 1$ mas collimation feature.

The limb brightened inner jet indicates transverse Doppler and/or emissivity structure. For uniform transverse emissivity, a jet sheath with $\beta_{s h}^{o b}=2$, so that $\gamma_{s h}=$ 2.45 , that is twice as bright as a lesser Doppler boosted jet spine requires a spine Lorentz factor of $\gamma_{s p} \sim 10$. This lies within the optical motion upper limit of $\gamma=10.5$ at HST-1 [145]. Tavecchio \& Ghisellini [158] were able to produce reasonable fits to the M 87 SED using comparable spinesheath Lorentz factors and viewing angle. Edge brightening could also be due to magnetic field generation and particle acceleration associated with KKHI in the velocity shear and also a dynamical effect as matter piles up along the edges of a collimating overpressured jet [159]. Most likely all of these effects contribute to the edge brightening observed along the inner and kpc jet.

The rapid variation in jet width and speed in the innermost 7 mas is sufficient to stabilize growth of global CDI or KHI inside about 2 pc. Still conversion of Poynting flux to kinetic flux could occur on unresolved spatial scales. Beyond about $2 \mathrm{pc}$ the jet expands more slowly and VLBI observations shown in Figure 11 [155] indicate twisted structure beyond $2 \mathrm{pc}$ that suggests growth of global CDI and/or KHI. Beyond the inner 7 mas the M 87 jet slowly collimates out to HST-1 and then expands with nearly constant opening angle out to knot $\mathrm{A}$ at kpc scales

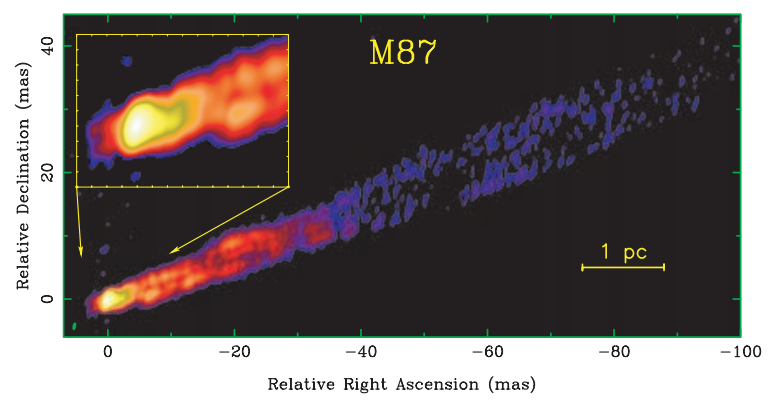

Figure 11. VLBA image of the M 87 jet at $15 \mathrm{GHz}$ with $\sim 1$ mas resolution. Inset enlarges the core region to show the inner jet and counter-jet [155]. Length scale is in the sky plane.

$[160,161]$ (Hada, this proceedings). There is evidence for twisted filaments along the 2 parsec to kiloparsec jet [157] and the filaments are consistent with a saturated KH elliptical mode on a decelerating jet flow from HST-1 to knot A [148], i.e., few $100 \mathrm{pc}$ to few kpc. Along with optical and radio motions at HST-1, optical and radio polarization observations along the kpc jet $[142,147]$ suggest that the M 87 jet has a spine sheath structure and organized flow and magnetic fields possibly out to knot $\mathrm{A}$.

\subsection{Tev Flaring, VLBA Monitoring \& Implications}

During 50 nights between January and May 2008 H.E.S.S., MAGIC and VERITAS found day scale variability in the energy range between 0.1 and 10 's of $\mathrm{TeV}$, and the flux reached the highest level ever observed from M 87 , $>10 \%$ of the Crab Nebula. At the same time the VLBA observed M 87 at $43 \mathrm{GHz}$ every 5 days. An initial radio flux density increase was located in the unresolved core. The radio peak occurred about 50 days after the onset of the VHE flare. Over this time the jet base brightened and extended about 0.77 mas, implying an apparent velocity of $1.1 \mathrm{c}$, and Lorentz and Doppler factors of $\sim 1.8$ and $\sim 2.8$, respectively at viewing angle $\theta=15^{\circ}[162]$.

$\mathrm{TeV}$ gamma-ray photons can escape the central region of M 87 without being heavily absorbed through $e^{+} e^{-}$pair production $[163,164]$ with either photons from the accretion disk [146] or infrared photons [165]. One can assume that the VHE flare is directly associated with the ejection event. As the disturbance propagates down the jet we see the radio rise later as a result of synchrotron self-absorption effects. Here the X-ray flux can rise and fall with the VHE if it comes directly from the disturbance. The TeV variability requires relatively small acceleration and emission regions. The radio structural change along with the timing of the VHE activity, imply that the VHE emission came from a region closer to the central engine than the VLBA resolution along the jet, $\sim 230 R_{S}$. The day scale variability implies an intrinsic timescale, $\Delta t_{\text {int }}<<\delta_{\text {max }} \Delta t_{\text {obs }} \sim 15 \times 10^{5} \mathrm{sec}$, and acceleration and emission region size $<<25 R_{S}=4.5 \times 10^{16} \mathrm{~cm}=c \Delta t_{\text {int }}$ where $\delta_{\text {max }}=2 \gamma$ with $\gamma=7.5$.

One possibility is vacuum gap electric field particle acceleration in the black hole magnetosphere where the $\mathrm{TeV}$ emission can be explained as IC emission of ultrarelativistic $e^{+} e^{-}$pairs produced in an electromagnetic cascade 
[163]. With acceleration and emission processes occurring on spatial scales on the order of $R_{S}$, the day scale TeV variability that requires $c \Delta t \sim R_{S}$ for a stationary acceleration and emission region is satisfied.

Another possibility is that the $\mathrm{TeV}$ emission comes from a fast moving flow at $<7^{\circ}$ to the line of sight near the jet base by IC scattering of low-frequency photons from a slower moving part of the jet. The rapid time variability requires small scale structures within the jet. CDI and reconnection timescales on the order of 100 Alfvén-light crossing times [166], require particle acceleration on spatial scales $<<25 R_{S} / 100$. Lenain et al. [167] showed that the VHE emission could come from small blobs $\left(\sim 10^{14} \mathrm{~cm}\right)$ radiating at distances just beyond an Alfvén surface located at $\sim 100 R_{S}$.

Needles-in-a-jet or jet-in-a-jet scenario high Lorentz factor structures lying within a slower moving flow could provide the small required spatial scales inside $230 R_{S}$. Where the flow is sub-Alfvénic, CDI driven reconnection particle acceleration can provide conversion of Poynting flux to plasma energy flux and the emission. Where the flow is super-Alfvénic, shock and velocity shear particle acceleration also can provide conversion of plasma energy flux and along with the background radiation field [168] could lead to a rapid deceleration and produce the emission in a deceleration zone [169].

In all of these scenarios the large scale jet spine Lorentz factor beyond $230 R_{S}$ need not significantly exceed the Lorentz factor at HST-1 and shocks and velocity shears on pc to kpc scales provide the sites for the particle acceleration and magnetic field generation indicated by the radio edge brightening [142], and by the optical and $\mathrm{X}$-ray knot emission.

\section{Summary}

The $\mathrm{mm}$ radio core in $\mathrm{M} 87$ is nearly coincident with the central engine. In general, emission from the inner M 87 jet can be modeled in the framework of moderate beaming so we are not blinded by strongly beamed emission coming from a large range of distances along a jet oriented close to the line of sight. The non-thermal synchrotron emission from radio to X-rays, the gamma-ray emission detected by Fermi/LAT, and TeV emission detected by the VERITAS, H.E.S.S., and MAGIC collaborations and the VLBA/VLA radio images of the $M 87$ jet have significant implications for particle acceleration and magnetic field generation processes at microscopic scales and for jet dynamics and stability at macroscopic scales.

On the M 87 jet, Poynting flux to plasma energy flux conversion and rapid particle acceleration almost certainly occur in the acceleration and collimation region at scales less than a few hundred gravitational radii from the black hole. The role of CDI and reconnection in Poynting flux to plasma energy flux conversion and particle acceleration at small scales and the role of shocks and velocity shear particle acceleration at small scales is not yet determined. The relative importance will depend on the sub-structure of the magnetic and velocity field. At larger scales the velocity shear between jet spine, sheath and ambient plasmas along with velocity or overpressure induced jet shocks provide sites for particle acceleration along the jet edge and in the jet interior via microscopic filamentation, KKHI and reconnection processes.

On relativistic jets global CDI and KHI can produce twisted global structures mixed with structure induced by non-uniformity of the flow. The roles of global CDI and KHI depend on: (1) the large scale transverse structure of the magnetic and velocity field, (2) the gradients associated with jet acceleration and expansion, and (3) the Lorentz factor and magnetosonic Mach number of the flow. On the M 87 jet global CDI and KHI are suppressed within the innermost few parsecs by the gradients associated with jet acceleration and expansion and likely also by the transverse structure.

Global CDI and KHI can develop beyond the innermost few parsecs to lead to the observed twisted filamentary structure from parsec to kpc scales. Here stabilizing influences must come into play in order to allow the jet to reach knot $\mathrm{A}$ at several kpc without jet disruption. Organized equipartition or higher helical magnetic field structure would be sufficient to suppress mixing over these length scales. A fully self-consistent magnetic CDI driven twisted filament model has not yet been developed. On the other hand, a self-consistent purely fluid KHI model can explain the basic behavior where stabilizing influences are primarily associated with jet expansion induced saturation from HST-1 to knot A. Ultimate destabilization at knot A results from shock heating and accompanying jet deceleration beyond HST-1 leading to trans-sonic speeds at knot A [148]. The true situation on scales from HST-1 to knot A is likely more complex with global spine-sheath and helical magnetic field structure within a complex cocooning medium playing a significant role in the stabilization/destabilization process.

\section{Acknowledgements}

P. Hardee acknowledges support from NASA NNX08AG83G, NNX12AH06G and NSF AST-0908010, and research collaborations with Dr. Ken-Ishi Nishikawa, Dr. Yosuke Mizuno, Dr. R. Craig Walker, Dr. Jean Eilek, Dr. Jose-Luis Gómez, Dr. Manolo Perucho and many others over the past years too numerous to mention individually.

\section{References}

[1] Gómez, J.L. et al. 2001, ApJ, 561, L161 (2001)

[2] Lobanov, A.P. \& Zensus, J.A. 2001, Science, 294, 128 (2001)

[3] Lobanov, A., Hardee, P., \& Eilek, J., New Science Reviews, 47, 629 (2003)

[4] Lery, T., Baty, H., \& Appl, S., A\&A, 355, 1201 (2000)

[5] Nakamura, M., Uchida, Y., \& Hirose, S., New Astronomy, 6, 61 (2001)

[6] Ouyed, R., Clarke, D.A., \& Pudritz, R.E., ApJ, 582, 292 (2003)

[7] Nakamura, M., \& Meier,D.L., ApJ, 617, 123 (2004)

[8] Nakamura, M., Li, H., \& Li, S., ApJ, 656, 721 (2007) 
[9] Moll, R., Spruit, H. C., \& Obergaulinger, M., A\&A, 492, 621 (2008)

[10] Moll, R., A\&A, 507, 1203 (2009)

[11] McKinney, J.C., \& Blandford, R.D., MNRAS, 394, L126 (2009)

[12] Carey, C.S. \& Sovinec, C.R., ApJ, 699, 362 (2009)

[13] Hardee, P.E., Clarke, D.A., \& Rosen, A., ApJ, 485, 533 (1997)

[14] Hardee, P.E., Hughes, P.A., Rosen, A., \& Gomez, E.A., ApJ, 555, 744 (2001)

[15] Hardee, P.E., \& Hughes, P.A., ApJ, 583, 116 (2003)

[16] Begelman, M. C., Blandford, R.D., \& Rees, M.J., Nature, 287, 307 (1980)

[17] Marscher, A. et al., Nature, 452, 966 (2008)

[18] Marscher, A. et al., ApJ, 710, L126 (2010)

[19] Sikora, M., Begelman, M., \& Rees, M., ApJ, 421, 153 (1994)

[20] Ghisellini, G., Celotti, A., Fossati, G., Maraschi, L., \& Comastri, A., MNRAS, 301, 451 (1998)

[21] Sikora, M., Blazejowski, M., Begelman, M.C., \& Moderski, R., ApJ, 554, 1 (2001)

[22] Dermer, C. et al., ApJ, 692, 32 (2009)

[23] Böttcher, M., \& Dermer, C., ApJ, 711, 445 (2010)

[24] Katarzynski, K., Sol, H., \& Kus, A., A\&A, 410, 101 (2003)

[25] Konopelko, K. et al., ApJ, 597, 851 (2003)

[26] Henri, G., \& Saugé, L., ApJ, 640, 185 (2006)

[27] Lister, M., ASPCS, 350, (ASP, San Francisco, 2006), 139

[28] Piner, B., Pant, N., \& Edwards, P., ApJ, 678, 64 (2008)

[29] Georganopoulos, M. \& Kazanas, D., ApJ, 594, L27 (2003)

[30] Ghisellini, G. et al., A\&A, 432, 401 (2005)

[31] Henri G. et al., Astroparticle Physics II, 347 (1999)

[32] Tammi, J. \& Duffy, P., MNRAS, 393, 1063 (2009)

[33] Stawarz, L., \& Ostrowski, M., ApJ, 578, 763 (2002)

[34] Blandford, R., \& Znajek, R., MNRAS, 179, 433 (1977)

[35] Krawczynski, H., ApJ, 659, 1063 (2007)

[36] Sikora, M. et al., ApJ, 625, 72 (2005)

[37] Istomin Y., \& Sol, H., Ap\&SS, 321, 57 (2009)

[38] Levinson, A., ApJ, 671, L29 (2007)

[39] Begelman, M.C., \& Fabian, A.C., \& Rees, M.J., MNRAS, 384, L19 (2008)

[40] Ghisellini, G., \& Tavecchio, F., MNRAS, 386, L28 (2008)

[41] Giannios, D., \& Uzdensky, D.A., \& Begelman, M.C., MNRAS, 395, 29 (2009)

[42] Komissarov, S.S., MNRAS, 359, 801 (2005)

[43] McKinney, J.C., ApJ, 630, L5 (2005)

[44] Meier, D.L., Ap\&SS, 300, 55 (2005)

[45] Blandford, R.D. \& Payne, D.G., MNRAS, 199, 883 (1982)

[46] Hawley, J.F., \& Krolik, J.H., ApJ, 614, 103 (2006)

[47] McKinney, J.C., MNRAS, 368, 1561 (2006)
[48] Mizuno, Y. et al., ApJ, 672, 72 (2008)

[49] Aloy, M., \& Mimica, P., ApJ, 681, 84 (2008)

[50] Begelman, M.C., ApJ, 493, 291 (1998)

[51] Spruit, H., Foglizzo, T., \& Stehle, R., MNRAS, 288, 333 (1997)

[52] Lyubarskii, Y.E., Sov.Astron.Lett., 18, 356 (1992)

[53] Lyubarskii, Y.E., MNRAS, 308, 1006 (1999)

[54] Giannios, D., \& Spruit, H.C., A\&A, 450, 887 (2006)

[55] Vlahakis, N., ApJ, 600, 324 (2004)

[56] Vlahakis, N., \& Königl, A., ApJ, 596, 1104 (2004)

[57] Vlahakis, N., \& Königl, A., Ap\&SS, 293, 67 (2004)

[58] Beskin, V.S., \& Nokhrina, E.E., MNRAS, 367, 375 (2006)

[59] Komissarov, S. et al., MNRAS, 380, 51 (2007)

[60] Levinson, A., \& van Putten, M., ApJ, 488, 69 (1997)

[61] Levinson, A., ApJ, 507, 145 (1998)

[62] Heinz, S., \& Begelman, M.C., ApJ, 535, 104 (2000)

[63] Hardee, P.E., ApJ, 664, 26 (2007)

[64] Mizuno, Y., Hardee, P., \& Nishikawa, K-I., ApJ, 662, 835 (2007)

[65] Istomin, Y. N. \& Pariev, V.I., MNRAS, 267, 629 (1994)

[66] Istomin, Y.N. \& Pariev, V.I., MNRAS, 281, 1 (1996)

[67] Narayan, R., Li, J., \& Tchekhovskoy, A., ApJ, 697, 1681 (2009)

[68] Bateman, G., MHD Instabilities, (MIT Press, Cambridge, Mass., 1978), 270

[69] Tomimatsu, A., Matsuoka, T., \& Takahashi, M., Phys.Rev.D, 64, 123003 (2001)

[70] Bodo, G., Mamatsashvili, G., \& Mignone, A., MNRAS, 434, 3030 (2013)

[71] Nalewajko, K., \& Begelman, M.C., MNRAS, 427, 2480 (2012)

[72] Nakamura, M., Uchida, Y., \& Hirose, S., New Astronomy, 6, 61 (2001)

[73] Mignone, A., Rossi, P., Bode, G., Ferrari, A., \& Massaglia, S., MNRAS, 402, 7 (2010)

[74] Porth, O., MNRAS, 429, 2482 (2013)

[75] Mizuno, Y., Lyubarsky, Y., Nishikawa, K.-I., \& Hardee, P. E., ApJ, 700, 684 (2009)

[76] Carey, C.S. \& Sovinec, C.R., ApJ, 699, 362 (2009)

[77] Mizuno, Y., Hardee, P., \& Nishikawa, K.-I., ApJ, 734, 19 (2011)

[78] Appl, S., Lery, T., \& Baty, H., A\&A, 355, 818 (2000)

[79] Baty, H., A\&A, 430, 9 (2005)

[80] Lery, T., Baty, H., \& Appl, S., A\&A, 355, 1201 (2000)

[81] Baty, H. \& Keppens, R., ApJ, 580, 800 (2002)

[82] Walg, S., Achterberg, A., Markoff, S., Keppens, R., \& Meliani, Z., MNRAS, 433, 1453 (2013)

[83] Birkinshaw, M. 1991, Beams and Jets in Astrophysics, (UCP, Cambridge, 1991), 278

[84] Ferrari, A., ARA\&A, 36, 539 (1998)

[85] Hardee, P.E., ApSS, 293, 117 (2004)

[86] Hardee, P.E., AIP Conf. Series, 856, (AIP, Melville, 2006), 57 
[87] Trussoni, E. 2008, Lect. Notes Phys., 754, (Springer, Berlin, 2008), 105

[88] Trussoni, E. 2009, Protostellar Jets in Context, (Springer, Berlin, 2009), 285

[89] Keppens, R., Meliani, Z., Baty, H., \& van der Holst, B., Lect. Notes Phys., 791, 179 (2009)

[90] Ferrari, A., Trussoni, E., \& Zaninetti, L., A\&A, 64, 43 (1978)

[91] Hardee, P.E., ApJ, 234, 47 (1979)

[92] Birkinshaw, M., MNRAS, 208, 887 (1984)

[93] Hardee, P.E., ApJ, 318, 78 (1987)

[94] Hardee, P.E., ApJ, 533, 176 (2000)

[95] Birkinshaw, M., MNRAS, 252, 73 (1991)

[96] Bodo, G., Rosner, R., Ferrari, A., \& Knoblock, E., ApJ, 470, 797 (1996)

[97] Ferrari, A., Trussoni, E., \& Zaninetti, L., MNRAS, 193, 469 (1980)

[98] Ferrari, A., Trussoni, E., \& Zaninetti, L., MNRAS, 196, 105 (1981)

[99] Ferrari, A., \& Trussoni, E., MNRAS, 125, 179 (1983)

[100] Ray, T.P., MNRAS, 196, 195 (1981)

[101] Londrillo, P., A\&A, 145, 353 (1985)

[102] Trussoni, E., Massaglia, S., Bodo, G., \& Ferrari, A., MNRAS, 234, 539 (1988)

[103] Hardee, P.E., ApJ, 664, 26 (2007)

[104] Cohn, H., ApJ, 269, 500 (1983)

[105] Feidler, R., \& Jones, T.W., ApJ, 283, 532 (1984)

[106] Appl, S., \& Camenzind, M., A\&A, 256, 354 (1992)

[107] Perucho, M., Hanasz, M., Martí, J.M., \& Sol, H., A\&A, 427, 415 (2004)

[108] Perucho, M., Martí, J.M., \& Hanasz, M., A\&A, 427, 431 (2004)

[109] Mizuno, Y., Hardee, P., \& Nishikawa, K-I., ApJ, 662, 835 (2007)

[110] Hardee, P.E., Rosen, A., Hughes, P.A., \& Duncan, G.C., ApJ, 500, 599 (1998)

[111] Hardee, P.E., Hughes, P.A., Rosen, A., \& Gomez, E.A., ApJ, 555, 744 (2001)

[112] Agudo, I. et al., ApJL, 549, L183 (2001)

[113] Hardee, P.E., \& Hughes, P.A., ApJ, 583, 116 (2003)

[114] Ferrari, A., Massaglia, S., \& Trussoni, E., MNRAS, 198, 106 (1982)

[115] Ray, T.P., MNRAS, 198, 617 (1982)

[116] Urpin, V., A\&A, 385, 14 (2002)

[117] Perucho, M., Martí, J.M., \& Hanasz, A\&A, 443, 863 (2005)

[118] Perucho, M., Hanasz, M., Martí, J.M., \& Miralles, J.A., Phys.Rev.E, 75, 631 (2007)

[119] Rosen, A., Hardee, P.E., Clarke, D.A., \& Johnson, A., ApJ, 510, 136 (1999)

[120] Xu, J., Hardee, P., \& Stone, J., ApJ, 543, 161 (2000)

[121] Frank, A., Jones, T.W., Ryu, D., \& Gaalaas, J.B., ApJ, 460, 777 (1996)

[122] Jones, T.W., Gaalaas, J.B., Ryu, D., \& Frank, A., ApJ, 482, 230 (1997)
[123] Keppens, R., \& Tóth, G., Phys.Plasmas, 6, 1461 (2000)

[124] Ryu, D., Jones, T.W., \& Frank, A., ApJ, 545, 475 (2000)

[125] Levinson, A., Phys. Rev. Lett., 85, 912 (2000)

[126] Maraschi, L., \& Tavecchio, F., ApJ, 593, 667 (2003)

[127] Kundt, \& Gopal-Krishna, J.Astrophys\&Astron., 25, 115 (2004)

[128] Spitkovsky, A., ApJ, 682, L5 (2008)

[129] Nishikawa et al., ApJL, 697, L1 (2009)

[130] Bret, A., ApJ, 699, 990 (2009)

[131] Nakar, E., Bret, A. \& Milosavljević, M., ApJ, 738, id.93 (2011)

[132] Sironi, L., \& Spitkovsky, A., ApJ, 698, 1523 (2009)

[133] Sironi, L., \& Spitkovsky, A., ApJL, 707, L92 (2009)

[134] Nishikawa, K.-I. et al., Adv.Sp.Res., 47, 1434 (2011)

[135] Nishikawa, K.-I. et al., IJMP Conference Series, 8, 259 (2012)

[136] Sironi, L., \& Spitkovsky, A., ApJ, 726, id.75 (2011)

[137] Sironi, L., Spitkovsky, A. \& Arons, J., ApJ, 771, id.54 (2013)

[138] Sironi, L. \& Spitkovsky, A., ApJ, 741, id.39 (2011)

[139] Alves, E.P. et al., ApJL, 746, L14 (2012)

[140] Liang, E., Boettcher, M., \& Smith, I., ApJL, 766, L19 (2013)

[141] Nishikawa, K.-I. et al., Ann.Geophys., 31, 1535 (2013)

[142] Owen, F.N., Hardee, P.E., \& Cornwell, T.J., ApJ, 340, 698 (1989)

[143] Sparks, W.B., Biretta, J., \& Macchetto,F., ApJ, 473, 254 (1996)

[144] Madrid, J.P. et al., Astrophys. Space Sci., 311, 329 (2007)

[145] Biretta, J.A., Sparks, W.B., \& Macchetto, F., ApJ, 520, 621 (1999)

[146] Cheung, C.C., Harris, D.E. \& Stawartz, L., ApJ, 662, 213 (2007)

[147] Perlman, E.S. et al., ApJ, 117, 2185 (1999)

[148] Hardee, P.E., \& Eilek, J.A., ApJ, 735, 61 (2011)

[149] Perlman, E.S., \& Wilson, A.S., ApJ, 627, 140 (2005)

[150] Mei, S. et al., ApJ, 655, 144 (2007)

[151] Gebhardt, K., ApJ, 729, 119 (2011)

[152] Walker, R. et al., JPhCS, 131, 012053 (2008)

[153] Walker, R. et al., ASPCS, 402, (ASP, San Francisco, 2009), 227

[154] Ly, C., Walker, R.C., \& Junor, W., ApJ, 660, 200 (2007)

[155] Kovalev, Y.Y., Lister, M.L., Homan, D.C., \& Kellermann, K.I., ApJ, 668, L27 (2007)

[156] Junor, W., Biretta, J.A., \& Livio, M., Nature, 401, 891 (1999)

[157] Lobanov, A., Hardee, P., \& Eilek, J., New Astronomy Reviews, 47, 629 (2003) 
[158] Tavecchio, F., \& Ghisellini, G., MNRAS, 385, L98 (2008)

[159] Zakamska, N.L., Begelman, M.C., \& Blandford, R.D., ApJ, 679, 990 (2008)

[160] Asada, K., \& Nakamura, M., ApJL, 745, L28 (2012)

[161] Hada, K. et al., ApJ, 775, id.70 (2013)

[162] Acciari, V. et al., Science, 325, 444 (2009)
[163] Neronov, A., \& Aharonian, F., ApJ, 671, 85 (2007) [164] Rieger, F., \& Aharonian, F., IJMPD, 17, 1569 (2008)

[165] Perlman, E. et al., ApJ, 663, 808 (2007)

[166] Mizuno, Y. et al., ApJ, 700, 684 (2009)

[167] Lenain, J.-P. et al., A\&A, 478, 111 (2008)

[168] Levinson, A., ApJ, 671, L29 (2007)

[169] Georganopoulos, M. et al., ApJ, 634, L33 (2005) 